\title{
A development strategy to fast establish the Taqman qPCR based method to detect SNP mutations
}

\author{
Xiaohui Jiang $^{1,2} \cdot$ Junbei Xiang ${ }^{3} \cdot$ Ruifeng Wang $^{4} \cdot$ Qian Wan $^{4}(\mathbb{C}$
}

Received: 15 April 2020 / Accepted: 6 June 2020 / Published online: 1 July 2020

(c) Japan Human Cell Society 2020

Dear Sir,

For the traditional Taqman qPCR based detection method to detect SNP mutations, the general idea is to run multiplex PCR or contain several probe pairs into one reaction tube, which can save material resources but may cost much time and energy [1-3]. The above idea is suitable under the normal conditions. However, it is unsuitable under the emergency conditions such as in the current COVID-19 pandemic, when the time efficiency is vital.

This study tried to develop the strategy to fast build the Taqman qPCR based detection method to detect SNP mutations in order that time could be saved or be gained with the cost of relatively ample material resources.

The novelty of this study was that by omitting of optimization for multiplex PCR and/or several probe pairs in one reaction tube, the method development time was saved or gained with the cost of relatively abundant material resources.

In this study, the investigation process was the following: (1) $1 \mathrm{ml}$ finger blood was collected from the individuals and the genomic DNA was extracted using the extraction kit (DP348, Tiangen Co. Ltd, China); (2) the human SNP site rs4148323 was selected as the experimental object because of the availability of related experimental materials and the

Qian Wan

129841480@qq.com

1 Human sperm bank, West China Second University Hospital of Sichuan University, Chengdu 610041, Sichuan, People's Republic of China

2 Key Laboratory of Birth Defects and Related Diseases of Women and Children (Sichuan University), Ministry of Education, Chengdu 610041, Sichuan, People's Republic of China

3 Sichuan Nursing Vocational College, Chengdu 610000, Sichuan, People's Republic of China

4 Chengdu Neo-Life Hope Medical Lab. Co. Ltd, Chengdu 610036, Sichuan, People's Republic of China richness of background information. The flanking sequence of rs4148323 was the following (the polymorphic site [G/A] was marked bold):

CAGCCACTGGCTGAGCATGCTTGGGGCCATCCA GCAGCTGCAGCAGAGGGGACATGAAATAGTTGTCCT AGCACCTGACGCCTCGTTGTACATCAGAGAC

\section{[G/A]}

GAGCATTTTACACCTTGAAGACGTACCCTGTGC CATTCCAAAGGGAGGATGTGAAAGAGTCTTTTGTTA GTCTCGGGCATAATGTTTTTGAGAATGATTC

(3) the primers and probes were designed with the software Primer Express 3.1 and synthesized by the local merchant (Qingke Co. Ltd). The sequences of the primers and probes are listed in Table 1. For the probes, they were ligated with the fluorescent molecule FAM at 5' end and the fluorescent quencher molecule TAMRA at 3' end; (4) Taqman qPCR: The $2 \times$ master mix for Taqman qPCR (SGExcel GoldStar) was purchased from Shanghai Sangon Biotech Co. Ltd. The qPCR instrument was Stepone Plus (ABI Co. Ltd, USA). For every detection, two tubes were prepared: in the first tube, $10 \mu \mathrm{l} 2 \times$ master, $1 \mu \mathrm{ls}-\mathrm{F}-1(2 \mathrm{OD} / \mathrm{ml}), 1 \mu \mathrm{l}$ rs-B-1 $(2 \mathrm{OD} / \mathrm{ml}), 2 \mu \mathrm{l}$ rs-Probe- $1(2 \mathrm{OD} / \mathrm{ml}), 6 \mu$ ultrapure water were added to give a total volume of $20 \mu \mathrm{l}$; in the second tube, $10 \mu \mathrm{l} 2 \times$ master, $1 \mu \mathrm{ls}-\mathrm{F}-1(2 \mathrm{OD} / \mathrm{ml}), 1 \mu \mathrm{l}$ rs-B-1 ( $2 \mathrm{OD} / \mathrm{ml}), 2 \mu \mathrm{l}$ rs-Probe-2 $(2 \mathrm{OD} / \mathrm{ml}), 6 \mu \mathrm{l}$ ultrapure water were added to give a total volume of $20 \mu \mathrm{l}$. The condition of Taqman qPCR was the following: $95^{\circ} \mathrm{C} 10 \mathrm{~min} ; 95^{\circ} \mathrm{C}$ $15 \mathrm{~s}, 60^{\circ} \mathrm{C} 60 \mathrm{~s}, 40$ cycles; (5) Sanger sequencing: Sanger sequencing was performed by the local merchant (Qingke Co. Ltd). The amplification primers were rs-F-1 and rs-B-1 and the sequencing primer was rs-F-1.

In result, in the first part, the heterozygote for rs 4148323 was detected and the results showed that the two reaction tubes both demonstrated smooth upward curves, so it could be judged that the sample was heterozygote for rs 4148323 (Fig. 1a, b), whose genotype was G/A.

Then, the above detection of heterozygote was verified with Sanger sequencing. The sequencing result showed the 
Table 1 The sequence information of primers and probes

\begin{tabular}{lll}
\hline ID & Name & Sequence(5' to 3') \\
\hline Primer 1 & rs-F-1 & TAGCACCTGACGCCTCGTT \\
Primer 2 & rs-B-1 & CACAGGGTACGTCTTCAAGGTGTA \\
Probe 1 & rs-Probe-1 & 5' FAM-TACATCAGAGACAGAGC-3' \\
& & TAMRA \\
Probe 2 & rs-Probe-2 & 5'FAM-TACATCAGAGACGGAGC- \\
& & 3'TAMRA
\end{tabular}

sample was the heterozygote for rs4148323, whose genotype was G/A (Fig. 1c). So, the new Taqman qPCR method for the SNP heterozygote was verified.

In the second part, the homozygote for rs4148323 was detected and the results showed that only the reaction tube with the probe rs-Probe- 1 demonstrated smooth upward curve and the reaction tube with the probe rs-Probe- 2 did not demonstrate smooth upward curve, so it could be judged that the sample was homozygote for rs4148323 (Fig. 2a, b), whose genotype was A/A.

The above detection of homozygote was verified with Sanger sequencing. The sequencing result showed the sample was the homozygote for rs4148323, whose genotype was a
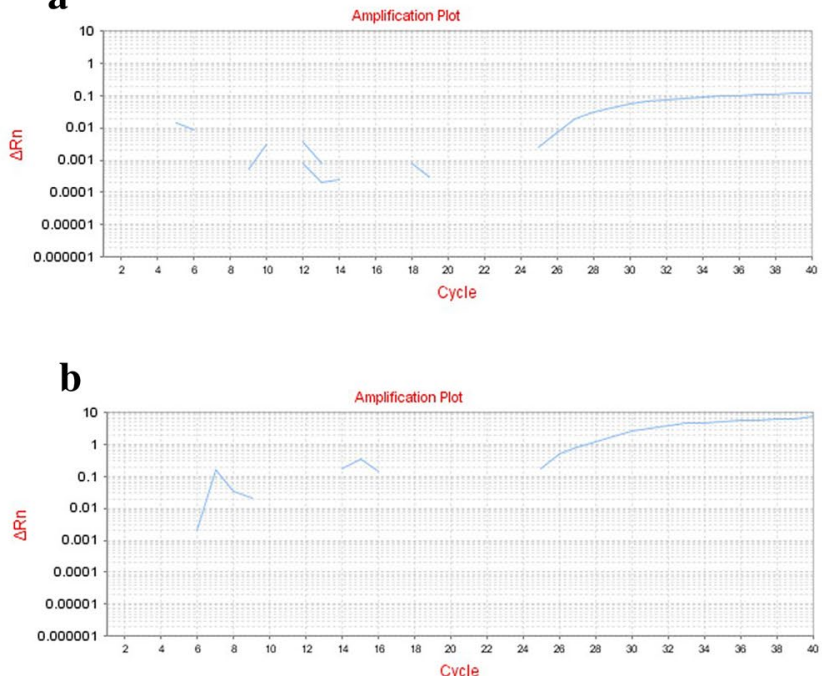

c

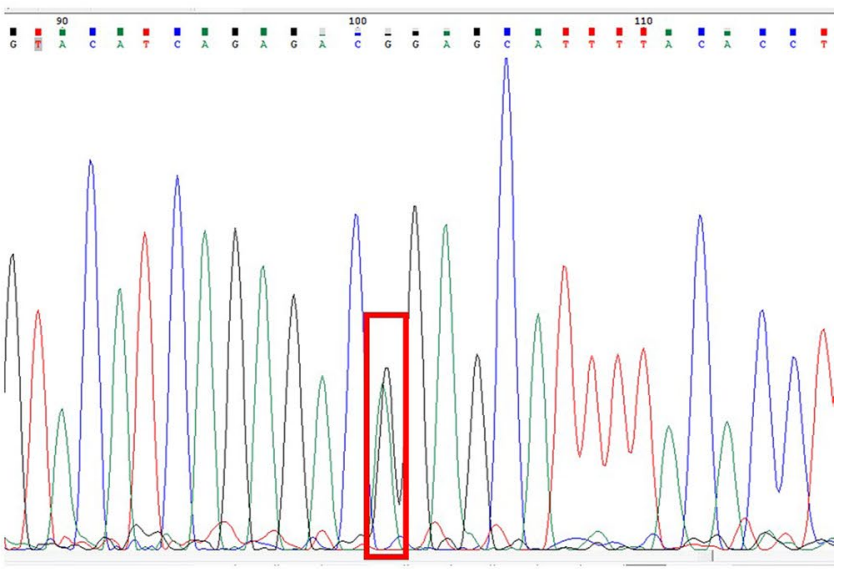

Fig. 1 The Taqman qPCR results and the verification by Sanger sequencing for the heterozygote sample for rs4148323. a The probe rs-Probe-1 was used; $\mathbf{b}$ the probe rs-Probe-2 was used; $\mathbf{c}$ the verification of new Taqman qPCR for the SNP hetero zygote by Sanger sequencing

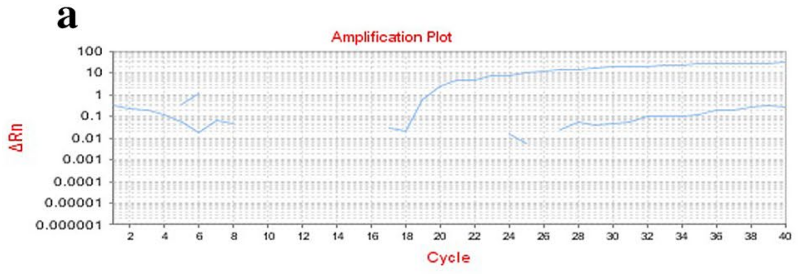

C

b
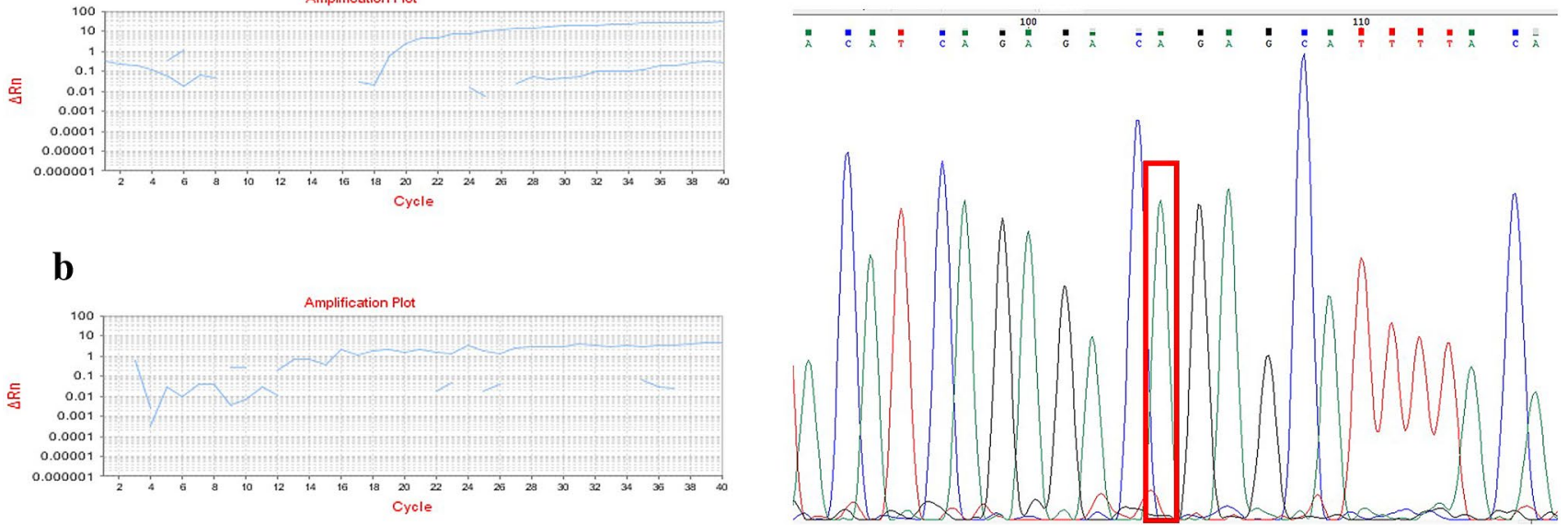

Fig. 2 The Taqman qPCR results and the verification by Sanger sequencing for the homozygote sample for rs4148323. a The probe rs-Probe-1 was used; $\mathbf{b}$ the probe rs-Probe- 2 was used; $\mathbf{c}$ the verification of new Taqman qPCR for the SNP homo zygote by Sanger sequencing 
A/A (Fig. 2c). So, the new Taqman qPCR method for the SNP homozygote was verified.

In conclusion, in this study, we developed a strategy to fast establish the Taqman qPCR method to detect SNP mutations. By the omitting of the optimization process of containing several primers and probes pairs in one reaction tube, the established strategy set up two reaction tubes, which was suitable for the emergency or time-limited conditions, such as for the current COVID-19 pandemic. The major novelty of this study was that by fullly exploiting the material resources, the development time of the detection method could be saved or gained.

Acknowledgements This study was supported by Program of Sichuan Health Commission of China (No. 16PJ404) and Deyang Science and Technology Project (No. 2018SZY072).

\section{Compliance with ethical standards}

Conflict of interest We declare that we have no financial and personal relationships with other people or organizations that can inappropriately influence our work. There is no professional or other personal interest of any nature or kind in any product, service, and/or company that could be construed as influencing the position presented in, or the review of, the manuscript entitled, "A development strategy to fast establish the Taqman qPCR based method to detect SNP mutations".

\section{References}

1. Tang Y, Shi C, Wu Z, Fan N, Xu X, Kang Z, Zhang X, Ma W, Guan M. A triplex probe-based TaqMan qPCR assay for Calreticulin type I and II mutation detection. Hematology. 2019;24(1):26-31.

2. Broeders S, Garlant L, Fraiture MA, Vandermassen E, Suin V, Vanhomwegen J, Dupont-Rouzeyrol M, Rousset D, Van Gucht S, Roosens N. A new multiplex RT-qPCR method for the simultaneous detection and discrimination of Zika and chikungunya viruses. Int J Infect Dis. 2020;92:160-70.

3. Pang Z, Li A, Li J, Qu J, He C, Zhang S, Li C, Zhang Q, Liang M, Li D. Comprehensive multiplex one-step real-time TaqMan qRT-PCR assays for detection and quantification of hemorrhagic fever viruses. PLoS ONE. 2014;9(4):e95635.

Publisher's Note Springer Nature remains neutral with regard to jurisdictional claims in published maps and institutional affiliations. 